\title{
e-Phaïstos
}

e-Phaïstos

Revue d'histoire des techniques / Journal of the history

of technology

IV-1 | 2015

Les arts de guerre et de grâce (XIVe-XVIIle siècles)

\section{L'expérimentation du geste martial et du geste artistique : regards croisés}

The testing of martial and artistic gestures: a cross analysis

\section{Daniel Jaquet et Dora Kiss}

\section{OpenEdition}

Journals

Édition électronique

URL : http://journals.openedition.org/ephaistos/657

DOI : 10.4000/ephaistos.657

ISSN : 2552-0741

Éditeur

IHMC - Institut d'histoire moderne et contemporaine (UMR 8066)

Édition imprimée

Date de publication : 1 avril 2015

Pagination : $56-72$

ISSN : 2262-7340

Référence électronique

Daniel Jaquet et Dora Kiss, «L'expérimentation du geste martial et du geste artistique : regards

croisés », e-Phaïstos [En ligne], IV-1 | 2015, mis en ligne le 23 novembre 2016, consulté le 08 novembre 2019. URL : http://journals.openedition.org/ephaistos/657 ; DOI : 10.4000/ephaistos.657 


\title{
L'expérimentation du geste martial et du geste artistique : regards croisés
}

\author{
Daniel Jaquet, \\ Département d'Histoire \\ Université de Genève \\ Dora Kiss \\ Institut de Recherche en Musicologie \\ CNRS, Paris
}

L'étude du geste a souvent été considérée comme secondaire par de nombreux courants historiographiques. La littérature technique conservée jusqu'à nos jours a été étudiée sous divers angles, comme par exemple celui de l'histoire de la pensée, ou de l'histoire des sciences et des techniques ; toutefois le geste qu'elle renferme, qu'il soit martial ou gracieux, a rarement constitué un objet d'étude à part entière. Nous proposons d'accorder dans cet article toute notre attention au geste, mais surtout de présenter la manière dont nous articulons recherche et expérimentation, en illustrant notre méthodologie par des exemples issus de nos objets d'étude respectifs' ${ }^{1}$.

Bien que les arts de guerre et de grâce puissent aujourd'hui paraître opposés, ils ont longtemps été étroitement liés ${ }^{2}$. Tant que la guerre reposait essentiellement sur l'homme, et à moindre titre sur les armes ou les machines, les apprentissages qui y préparaient impliquaient un enchaînement causal identique à ceux des arts de grâce : en effet, d'abord le corps du combattant doit être entraîné (au même titre que celui du danseur) ; puis cet entraînement transforme tout à la fois sa corporalité et l'art auquel s'adapte cette dernières.

\section{Approche de la corporalité : cadre théorique}

La représentation du geste, selon Guillemette Bolens ${ }^{4}$, est à approcher à l'aide d'outils bien spécifiques qui permettent de distinguer différentes facultés des trois ordres suivants : l'ordre physique, perceptif, et sensitif. D'ordre physique, «la kinétique renvoie aux mouvements matériels de n'importe quelle sorte de corps du point de vue des lois de la physique [...] ». D'ordre perceptif, la kinésie est « la perception chez autrui ou soi-même de mouvements en fonction de paramètres viuomoteurs, traduits en données telles que l'amplitude, l'extension ou la vitesse d'un geste [...] ». Elle permet une analyse, que Guillemette Bolens applique aux textes littéraires, qui « consiste à observer les moyens exacts choisis dans l'œuvre pour communiquer en faisant référence au mouvement corporel et en générant des simulations perceptives chez le destinataire du texte ". Cette "simulation relève d'actes cognitifs dynamiques, correspondant à des intentions de produire du sens, plutôt qu'à des produits idéals cernés, isolables et figés. » D'ordre sensitif, « la sensation kinesthésique est exclusivement propre à la personne qui la ressent ». Étant donné que nous développons une approche de nos sources 
par l'expérimentation, nous dégageons dans cet article la notion supplémentaire de "signification kinesthésique ». Cette dernière est intrinsèque au mouvement et dépendante de la sensation de ce dernier, a priori chez celui qui l'exécute. La signification kinesthésique apparaît par l'activation d'un mouvement ressenti dans toute sa portée signifiante sans que cette activation ait nécessairement pour but de communiquer une signification; ressentir cette signification peut être une fin en soi. Cela dit, à condition que cette signification soit saisie par l'agent du mouvement, elle peut potentiellement être communiquée soit par le partage de l'expérience du geste, soit par la simulation de ce dernier. Une expertise préalable dans la technique corporelle dont dépend le geste facilite tout à la fois ce partage et cette simulation, en particulier pour les gestes dont l'exécution nécessite un entraînement. Enfin, dès lors que la signification kinesthésique est communiquée, elle peut faire l'objet d'une analyse kinésique.

\section{Approche de la corporalité : enjeux}

Le corps, en termes philosophiques, facilite la transcendance, mais dans le contexte de notre article, il est avant tout une mécanique qu'il s'agit de former au service d'une pratique gestuelle. Le concept de corporalité quant à lui permet de valoriser le caractère du corps comme le tout de l'homme et comme informant la subjectivité et les comportements humains. Et en effet, ce n'est pas qu'en tant que squelettes soutenus par une masse musculaire que les aristocrates combattent ou dansent, mais en tant que totalités regroupant corps, âme, personnalité singulière et rôle social.

Le corps du combattant a toujours été façonné. L'identification des manières et des moyens de cette « mise en forme » est un travail qui nécessite pour l'historien un questionnement spécifique, s'adaptant à un corpus de sources complexe à constituer et à interroger. L'avènement du Prozess der
Civilisation 5 , qui examine le passage du chevalier médiéval au courtisan de la Renaissance, permet de reconstruire une identité corporelle, puisque, dès lors, le corps prend une place plus importante dans les écrits au même titre que dans les mentalités et les usages culturels. Toutefois, une autre méthode permet d'apporter des réponses : s'interroger sur la praxis, autrement dit sur les pratiques corporelles consignées, inscrites ou codifiées dans une littérature technique, en particulier, les gestes. Ainsi, il est possible d'entrevoir la corporalité du combattant à travers sa praxis spécifique, les gestes qui forment son corps et son "intelligence du corps ${ }^{6}$ par la pratique routinière ou exceptionnelle. Le chercheur entrevoit ainsi la corporalité du combattant par le prisme des gestes, mis en perspective par une textualité qui cherche à régler sa place et son façonnage pour correspondre à un idéal. La limite est bien là : le corps du guerrier est sélectionné, mais ce n'est pas son corps qui en fait un combattant, mais bien son instrumentalisation.

$\mathrm{Au}$ XVIII ${ }^{\mathrm{e}}$ siècle, le corps n'existe pas en tant qu'instrument du danseur; son usage repose sur l'idéal de naturel, et ne s'écarte théoriquement que peu d'un usage ordinaire - bien que les qualités telles que souplesse, force des membres inférieurs, légèreté et grâce soient requises, à plus forte raison pour danser que pour d'autres activités. On attend cependant certaines spécificités corporelles du danseur, qui ne sont pas toujours innées. Pour ce qui est de la posture par exemple, il faut qu'il se tienne droit et porte attention à son en-dehors (la position des pieds présentant un angle de quarante-cinq à soixante degrés). Par ailleurs, il faut qu'il possède une intelligence kinesthésique développée et complexe : c'est par elle qu'il peut combiner dextérité et bienséance, deux qualités qui dans leurs extrêmes peuvent s'opposer. C'est aussi grâce à elle qu'il est capable de placer ses gestes sur la musique - implicitement, il est censé également s'accorder avec son ou ses partenaires. Cette intelligence particulière se cultive tout autant que la souplesse. Le danseur, 
cependant, n'a pas seulement un corps. En effet, il est identifié à la personne ou au rôle qu'il incarne en tant qu'interprète ; il est donc une corporalité singulière et distinctive, culturellement et socialement. Par exemple, la tenue à laquelle nous faisions allusion départage le danseur (gentilhomme ou roturier) d'autres personnes moins éduquées ; et tout à la fois elle se confond pour certains aspects avec celle de l'homme d'armes. Sachant que le modèle du combattant, comme celui du- gentilhomme danseur, se construit d'après les représentations de la noblesse, cette " anatomie » commune de la droiture et d'une souplesse débouchant sur la rapidité des mouvements des membres inférieurs n'est pas un hasard ; bien au contraire, cette communauté est représentative d'un idéal moral de l'homme dont le corps est symptomatique7. En somme, le corps témoigne de l'incarnation d'une certaine nature intérieure.

Le modèle de l'homme noble est fier et droit, gracieux et leste, fût-il à la cour ou au combat. Et le danseur? Lorsqu'il n'est pas aristocrate et qu'il s'adonne à la " belle danse », il est probablement immanquablement professionnel; alors, il peut remplacer sur la scène les nobles dont l'habileté n'est pas ou plus adaptée aux nouvelles exigences techniques de sa mise en scène ; il peut aussi incarner, au théâtre, à l'opéra, au ballet, des héros qui eux-mêmes représentent symboliquement les nobles ${ }^{8}$. Bref, pour autant qu'il ne tienne le rôle ni d'un saltimbanque, ni d'un paysan, ni d'un Turc (la liste des « équivalences » pourrait s'allonger), alors ce danseur « noble » au sens de beau, d'élevé, appartient au petit nombre des figures auxquelles, corporellement du moins, on peut s'identifier'.

Durant l'Ancien Régime, tant l'investissement corporel idéal que la «conduite du corps » sont donc à la fois codifiés et partagés par plusieurs niveaux de la société. Mais les corps et leurs investissements, au sein d'une époque et d'une classe sociale, sont bien sûr distinctifs en fonction des arts exercés.
Aujourd'hui, les sources historiques relatives aux gestes évoquent donc des corporalités qui semblent bel et bien particulières, d'autant qu'elles sont différentes de celle de la personne qui consulte les sources «depuis » le XXI ${ }^{2}$ siècle. La distance temporelle de plusieurs siècles peut d'ailleurs être une condition favorable pour la perception de spécificités kinésiques et kinétiques propres à une époque, un art, un auteur ; ou encore un exemple spécifique de combat en armes ou de chorégraphie. Et pour tenter, précisément, de comprendre ces corporalités dans leurs singularités, les chercheurs développent des questionnements qui s'adaptent au type de sources interrogées.

\section{Sources : points communs et particularités}

Les sources relatives au combat en armure du $\mathrm{XV}^{\mathrm{e}}$ siècle et à la belle danse ont des points communs. Les textes qu'elles contiennent présentent des données techniques relatives au geste, et annoncent des projets auctoriaux qui ne paraissent pas toujours en accord avec leur contenu. Ainsi, elles invitent à une analyse double qui vise à comprendre le geste décrit d'une part, et le statut de cette description d'autre part. En plaçant la description dans le contexte d'un projet auctorial (les sources peuvent en effet relever de la transmission de savoirs, de la volonté de mise en mémoire, de la défense d'un art ou d'une technique, ou encore de la prise de parole d'un auteur dont la volonté peut être de corriger la pratique en cours), il est davantage évident de comprendre à quel type de geste on a affaire. Il peut s'agir de gestes pratiqués, normés, idéalisés, simplifiés...

En outre, ces sources textuelles, tout au moins celles dont le caractère est didactique ou prescriptif, contiennent bien souvent des images, dont il faut cette fois identifier le type de complémentarité avec le texte: s'agit-il d'une illustration de la posture préalable au geste, du geste lui-même ; l'image doitelle suppléer aux ellipses du texte, ou l'illustre-telle? 10 
On le voit : même pour des recherches de type fondamental, nos corpus inviteraient tous deux à une approche pluridisciplinaire. Cela dit, chacun de nos corpus a aussi sa singularité, et invite donc à d'autres approches.

Les sources relatives au combat de la fin du XIVe au début du XVIe siècle forment un corpus hétéroclite dans le fond comme dans la forme. Il est constitué de près de huitante manuscrits et d'une trentaine d'imprimés (1389-1570) ${ }^{11}$ traitant des gestes martiaux, divisés en trois catégories: le combat civil, le combat à cheval et le combat en armure à pied. Nous nous intéresserons ici à vingt-quatre manuscrits qui concernent directement le dernier de ces arts. La majeure partie est composée de texte et d'images, de qualité et de quantité différentes. Toutefois, ils ont tous un lien avec des formes d'affrontements normés (duels singuliers) pratiqués par des combattants en armure dans ce grand XVe siècle. Si les projets auctoriaux diffèrent (florilège technique, réduction en art, œuvre réalisée pour la postérité d'un maître, d'une cour ou d'un prince, etc.), le fond est relativement commun, inscrivant, codifiant ou décrivant les techniques personnelles de combat dans des situations agonales. Ces sources forment un sous-genre particulier de la littérature technique qu'il faut différencier des livres de tournois, livres de maison, livres de guerre ou encore livres de croquis, même si parfois les sous-genres sont connexes.

Les sources relatives à l'art de la danse du début du XVIII e siècle comportent une variété de traités importants, qui pour la plupart sont imprimés ${ }^{12}$. Elles sont également constituées de partitions chorégraphiques qui certes n'encodent que les déplacements des danseurs et certains de leurs mouvements des membres inférieurs, reste qu'elles documentent de manière exceptionnelle les savoirs corporels européens propres à la danse pour lesquels la transmission directe a été rompue. Ces partitions, découlant directement de la publication de $\mathrm{La} C h \mathrm{C}^{-}$ régraphie, ou l'art de décrire la danse (Raoul Auger
Feuillet, Paris, 1700) appartiennent aussi au champ de la musicologie tel qu'il est communément défini. En effet, il y apparaît, sur chaque page d'une danse notée, une portée musicale sur laquelle est inscrite une mélodie, support sonore à la chorégraphie. Cette mélodie renvoie à d'autres sources musicales dont la teneur peut contribuer à donner des informations sur le caractère d'une danse, sur son tempo, et donc sur le style de son exécution ${ }^{13}$. En résumé, les partitions chorégraphiques invitent non seulement à leur expérimentation, mais aussi à l'expérimentation des pièces musicales auxquelles elles se réfèrent.

Cependant, pour évaluer la compréhension des sources historiques relatives au combat du $\mathrm{XV}^{\mathrm{e}}$ et XVI ${ }^{\mathrm{e}}$ siècles ou à la danse du XVIII ${ }^{\mathrm{e}}$ siècle, se pose de façon aigüe la question de la validité des hypothèses que fera le chercheur à la consultation des sources, que cela regarde la corporalité ou les savoirs gestuels.

En ce qui concerne les gestes martiaux, sont-ils conçus pour tuer ou pour jouer ? Cette bipolarité (Schimpf und Ernst, ludique ou sérieuse ${ }^{14}$ ) nécessite d'être réduite afin de pouvoir établir une grille de validation des hypothèses, principalement déterminées par la finalité du geste. Évaluer un geste nécessite non seulement de comprendre son cadre d'application, mais aussi de reconstruire les critères contemporains de perception d'un geste "réussi ». $\mathrm{Si}$ la dimension esthétique entre également en compte, c'est surtout la dimension pragmatique qui est aisément mesurable (blessure, fracture, projection, etc.). Pour ce qui est des arts de grâce, les gestes n'émanent-ils pas avant tout d'un système esthétique, éminemment subjectif, dont les règles et l'application appartiennent si ce n'est au seul danseur, du moins à l'ensemble des artistes qui contribuent à élaborer une pièce chorégraphique ? La recherche dite scientifique ne permet probablement pas de retrouver une esthétique qui n'est plus en vigueur, et donc de savoir quel geste est en soit « réussi » ou « raté ». Elle peut cependant dégager le 
fonctionnement de ce système esthétique, et donner à sentir comment s'y organisent différents éléments pour l'élaboration d'un " discours ». La recherche pourrait aussi proposer quelques instruments pour départager ce qui relève d'une part des " faits chorégraphiques » et d'autre part de leur interprétation.

Que cela soit pour trouver le geste " réussi » ou pour comprendre le fonctionnement d'un système esthétique en vertu duquel le geste le plus juste possible pourra être déterminé, il convient d'être inventif, à notre avis, pour ce qui est de la méthodologie, et de renoncer à une approche trop strictement fondamentale.

Les sources de nos corpus ont, à ce titre, d'autres points communs : elles ont le grand intérêt de renseigner sur les aspects mécaniques de la réalisation des danses ou des combats. Elles ont néanmoins un double inconvénient: elles ne donnent pas toutes les clefs de la mécanique du mouvement et ne renseignent pas explicitement sur le potentiel respectivement pragmatique et expressif du mouvement. Autrement dit, si elles permettent un accès privilégié à certains aspects kinétiques d'un art et d'une culture, elles suscitent néanmoins passablement de doute: pouvons-nous nous faire aujourd'hui une représentation quant à la manière fine de réaliser les mouvements décrits ? Pour y parvenir, il s'agit pour le chercheur de réussir à identifier, puis à reconstruire les éléments implicites ou les savoirs préalablement requis pour la lecture et la réalisation des gestes. Alors seulement il est possible d'évaluer les résultats. Cette démarche, de fait, amène plusieurs questions. Comment réalisait-on concrètement ces mouvements, et dans quelle corporalité étaient-ils inclus ? Quel était leur «fonctionnement » interne, et en conséquence, quel ressenti déclenchait leur enchaînement chez le combattant ou le danseur ? La perception d'une séquence permettait-elle de situer sa performance par rapport à l'idéal d'une " gestuelle » - organisée en vertu d'un résultat vis-à-vis du partenaire ou du public ? D'autres questionnements appartiennent plus exclusivement au champ de la recherche en danse ou au combat. Par exemple pour la danse : quel était l'effet des gestes dansés sur le plan de l'affect, et comment cet effet était-il d'une part construit par les danseurs, d'autre part identifié par les spectateurs ? Est-ce qu'il s'agissait alors de prendre telle posture ou d'exécuter tel mouvement pour communiquer un sens kinesthésique visé, ou étaitce plutôt un assemblage (unique), un enchaînement (repris plusieurs fois), ou une séquence (convenue) sur lequel reposait ce sens ? Pour le combat: de quelle manière le geste était-il entraîné, puis performé ? Y avait-il une différence ? Si oui, comment le geste était-il pratiqué et évalué dans chacun des cas?

À travers toutes ces questions, il appartient finalement au chercheur de pouvoir définir quelles sont les questions particulières pour son objet de recherche, et quels sont les questionnements plus larges qui permettent d'appréhender les gestes dans leur ensemble. Le point de départ de notre article est le constat suivant : la seule étude documentaire ne permet pas d'aborder certains points qui nous semblent pourtant centraux.

\section{L'approche expérimentale}

"The use of weaponry engages the physical body as much as (if not more than) the intellect, and it is therefore essential for us to investigate them through bodily experience ${ }^{15}$ ", cette remarque essentielle concerne également les arts de grâce comme la danse. L'expérimentation est donc une des méthodes indispensables pour réduire ces questionnements, en complément aux méthodes plus habituelles. Elle doit aujourd'hui à notre sens être définie, en particulier pour tenter de la distinguer d'autres démarches qui sont menées en référence aux sources que nous consultons également, mais dans d'autres perspectives que celles de l'approche définie comme scientifique ${ }^{16}$. Au lieu de situer l'expérimentation gestuelle telle que nous la pratiquons par rapport à des sous-disciplines établies 
(archéologie expérimentale, ethno-archéologie, tracéologie, etc.) qui répondent toutes à des besoins particuliers liés à l'objet d'étude et à la méthodologie propre à la discipline à laquelle elle se rattache, il est intéressant d'établir nos besoins spécifiques en rapport avec les particularités de notre objet d'étude.

La définition de la démarche expérimentale, point d'aboutissement de la révolution scientifique entamée au XVIe siècle, remonte à la correspondance de Michel Eugène Chevreul (1856) ${ }^{17}$. Cette « nouvelle » approche fait bien entendu l'objet de perfectionnement, d'appropriation et de débats encore aujourd'hui. Les enjeux principaux sont la problématisation (inductive ou déductive), la nature des tests et les moyens d'évaluation ${ }^{18}$. Dans la mesure où la méthode élaborée correspond aux critères scientifiques établis, l'expérimentation gestuelle est à distinguer d'autres démarches souvent associées ou encore confondues, comme la reconstitution, la recréation ou la restitution. Elle diffère dans les enjeux (validation d'une hypothèse établie à partir d'une démarche scientifique), dans les moyens (tests et expérimentations établis par des protocoles) et dans la visibilité (publication). Il s'agit donc d'identifier les problèmes qui font obstacle à la compréhension (sens et technique) à partir des sources. Une fois formalisés, ces problèmes issus de l'analyse des sources de manière inductive, font l'objet d'établissement de différentes hypothèses par le chercheur via la rédaction d'un protocole d'expérimentation qui vise à tester les différents postulats afin de corroborer ou de réfuter ces derniers.

À ce titre, l'expérimentation gestuelle permet l'obtention de données et l'analyse de celles-ci à plusieurs niveaux. Nous en distinguons deux principaux à la lumière de nos travaux. Le premier est la réduction des abstractions textuelles et iconographiques. En effet, l'approche pluridisciplinaire de nos objets d'études permet d'apporter des éléments de réponse à une partie des problèmes, mais les supports physiques ont leurs limites que l'expérimentation permet de combler. L'abstraction identifiée peut être résolue par l'expérimentation gestuelle qui réfutera ou validera les postulats établis à partir du corpus de sources étudiées. Le deuxième niveau concerne l'intelligence kinesthésique. Les supports physiques n'abordent pas les questions essentielles pour le chercheur qui s'intéresse à des problématiques liées à la (re)découverte d'un savoir sensorimoteur. La majeure partie de ces questionnements ne trouve pas de réponse dans nos sources puisque ce savoir est un prérequis du lecteur qui peut être explicité ou non. Ainsi, l'expérimentation permet dans une certaine mesure d'aborder le domaine du sensible d'une kinésie historicisée.

Philippe Guisgand, lorsqu'il élabore une lecture des œuvres d'Anne Theresa de Kaersmaker, affirme :

« [...] ce livre s'adresse aux étudiants en danse et aux chercheurs de ce champ universitaire en développement. Dans le cadre d'étude en danse et non pas sur (le dos de) la danse -, il est incontournable et inhérent à cette discipline de donner aux mots la densité des gestes qu'ils décrivent. La danse nous arrive par le corps et je fais l'hypothèse que sa pratique amplifie cette perception spécifique (de même que j'admets volontiers qu'un musicien entend plus de choses que moi qui ne le suis pas). Cet avantage s'avère un des principaux postulats théoriques de cette lecture des œuvres pressentant - à partir de la description et l'analyse de la danse au sein des pièces - l'existence d'interprétations inexplorées et pourtant centrales. En effet, en tant que danseur, je me fraye à travers le syncrétisme d'une œuvre chorégraphique, un chemin naturel qui passe d'abord par l'observation du mouvement : l'art de la danse me touche donc par où je le côtoie. Cette interprétation à partir de la description de la danse me rapproche de la phénoménologie de Merleau-Ponty et de la lecture qu'en fait Sondra Horton Fraleigh. 19 »20

Pour l'auteur, il est évident que son savoir sensorimoteur est adéquat pour la «lecture »; il le démontre d'ailleurs par l'écriture d'une représentation l'œuvre contemporaine d'Anne Theresa de Kaers- 
maker, à laquelle on peut par ailleurs avoir notre propre accès. Nous ne pouvons pas nous prévaloir d'une telle assurance ; le caractère historique de notre savoir sensorimoteur n'est pas entièrement démontrable. La pratique de nos arts, à toutes sortes d'égards, peut néanmoins être éclairante.

Pour ce qui est de notre démarche, il ne s'agit pas, au premier contact avec les sources, de passer immédiatement à la pratique ; bien au contraire, il nous faut d'abord faire usage de notre simulation perceptive, quitte à repasser plusieurs fois par le texte, pour ne comprendre ne serait-ce que ce qui nous interpelle ou nous interroge. C'est à partir de cette simulation que nous pouvons conceptualiser intellectuellement la corporalité du danseur ou du combattant, une séquence de mouvements, ou un geste dans toute sa portée (causale, signifiante). Et pour cette lecture des sources primaires, nous ne pouvons pas uniquement nous référer à notre corporalité contemporaine. Au contraire, nous devons la mettre à distance, et nous référer plutôt à notre corps entraîné à nos arts de guerre ou de grâce, des corps qui à défaut d'être corporalités au même titre que celles des combattants et danseurs historiques, peuvent être des « médias » pour nos expérimentations. Pour comprendre, précisément, à quelle moment l'expérimentation intervient et à quoi elle sert, le lecteur sera accompagné dans notre processus, que nous n'illustrons que très partiellement par deux exemples pratiques issus de nos travaux, l'un concernant le combat, l'autre la danse.

\section{Expérimentation du geste guerrier}

L’exemple présenté ici illustre le premier niveau d'investigation, à savoir les abstractions textuelles et/ou iconographiques. Nous nous intéresserons à quatre manuscrits appartenant au groupe Gladiatoria $^{21}$, qui présente un certain nombre de ces abstractions. Ces livres de combat manuscrits regroupent le même traité avec des variantes. Ils traitent exclusivement du combat en armure à pied en relation avec des affrontements normés tels que pratiqués au XVe siècle (duel judiciaire, tournois ${ }^{22}$ ).

Nous nous intéresserons en particulier au manuscrit de Vienne ${ }^{23}$, proposant la version la plus complète. À l'intérieur de ce témoin, un concept spatial est mentionné plusieurs fois sans être introduit ou commenté par l'auteur. Les termes «intérieur » et " extérieur » 24 apparaissent vingt et une fois dans les commentaires techniques, souvent pour désigner un espace cible pour l'attaque initiale. Il apparaît clairement que ces deux concepts sont opposés et influent de manière mécanique sur les pièces techniques décrites. L'abstraction textuelle n'étant pas réduite de façon pertinente par l'analyse iconographique et celle de son lien avec les commentaires, l'abstraction persiste, comme le montre l'exemple suivant :

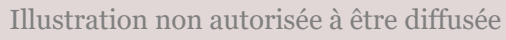

Figure 1 : Anonyme, Gladiatoria, 1425-145o. Kunsthistorisches Museum, Wien, KK5013, $f^{\circ} 13 r$. 


\begin{abstract}
"Lorsque tu l'estoques par en bas à l'intérieur de son épée au visage, alors saisis-toi de sa main gauche derrière le gantelet avec ta main gauche depuis ton épée et marche avec ton pied gauche à l'intérieur de son pied gauche. Fais levier avec ton épée sur son bras par-dessus lui. Lorsque tu l'as ainsi déséquilibré, alors laisse aller la main gauche de ton épée et saisis-rapidement par-delà la gorge et avec ta main gauche, attrape son épaule gauche à l'intérieur. Ainsi tu le projettes, comme tu le vois peint ci-dessus. » 25
\end{abstract}

En analysant l'ensemble des pièces, il apparaît que l'élément distinctif entre le concept d'intérieur et d'extérieur est l'épée tenue par l'adversaire. Il faut mentionner que cette saisie est spécifique au combat en armure au XVe siècle, il s'agit de la « demi-épée » ou « épée raccourcie ${ }^{26}$ qui consiste à saisir avec la main forte la poignée de l'épée et avec la main faible, le centre de lame, permettant ainsi une meilleure maniabilité, une efficacité renforcée de l'estoc et la possibilité d'utiliser la longueur de l'arme comme un levier de force, ainsi que les parties inférieures comme crochets ou éléments de percussion. Si l'on considère que l'élément distinctif est donc la pointe de l'épée adverse qui nous fait face, les deux postulats suivants doivent être expérimentés : l'intérieur est à droite de la pointe ou l'intérieur est à gauche de la pointe ${ }^{27}$.

Il est intéressant de noter ici que la démarche de l'expérimentateur doit absolument faire abstraction de tout savoir relatif à des pratiques formant un fond référentiel d'expériences identifiables, afin de ne pas induire d'éléments perturbateurs dans l'analyse. Par exemple, la pratique antérieure de sports ou d'arts modernes comme l'escrime induira en erreur l'expérimentateur, puisque ce concept est défini dans les pratiques sportives et ce depuis le XVIIe siècle. L'intérieur est compris comme "l'intérieur des armes », à savoir la partie du corps qui est le moins bien couverte par l'arme tenue à une main. Ceci ne correspond en rien à notre problème puisque d'abord, la tenue de l'arme diffère, mais surtout puisque ce type de pratiques normées de duel désarmé (sans armure) ne correspond absolument pas à l'objet de nos investigations.

Les deux postulats font donc l'objet d'expérimentations, consignées par protocoles écrits rédigés afin de compiler les différentes données, à partir de plusieurs pièces techniques sélectionnées ${ }^{28}$. Chacune des expérimentations se déroule selon quatre niveaux d'exécution, établis de manière à réduire les problématiques liées à l'équipement, la sécurité et le savoir sensorimoteur des expérimentateurs 29 .

Les données brutes de l'expérimentation sont analysées selon plusieurs critères de validation. Ceux-ci dépendent de la problématisation, mais de manière générale, les critères suivants sont examinés :

- Correspondance: Les séquences gestuelles expérimentées identifiables correspondent aux informations textuelles et iconographiques brutes de la source (données initiales) ;

- Finalité : S'il est possible d'identifier la finalité de la technique à partir des données initiales brutes (blessure létale ou incapacitante, projection, clé de soumission, désarmement, etc.), les résultats obtenus par l'expérimentation correspondent à cette finalité ;

- Système : Les techniques s’inscrivent la plupart du temps dans un système plus vaste dont il est possible de tirer des principes. Ces principes sont soit explicites, soit implicites. Dans le second cas, ils font l'objet d'hypothèses liées au cadre d'application de la technique ${ }^{30}$;

- Kinésie : De manière empirique, les séquences gestuelles ne doivent pas faire l'objet "d’incohérences biomécaniques ». Si les données initiales qui font l'objet d'une abstraction amènent les expérimentateurs à exécuter des mouvements " contre nature", ou provoquant des gênes (équipement), le postulat en question doit faire l'objet d'un réexamen ${ }^{31}$. 
L'objectif à terme est de corroborer ou de réfuter les postulats qui font alors l'objet d'une comparaison formelle. Il est possible que l'analyse ne permette pas de manière probante de sélectionner un seul postulat en écartant les autres, mais que plusieurs soient plausibles. Cela dépend de la problématisation à l'origine de l'expérimentation en question. Toutefois, pour l'exemple mentionné, un des postulats est clairement plausible, tandis que l'autre pose des problèmes ou conduit à des incohérences. En l'occurrence, la phase d'expérimentation gestuelle permet de valider le postulat qui place l'intérieur à droite de la pointe de l'épée adverse.

\section{Expérimentation du geste de danse}

Nous tirerons notre exemple de geste dansé du traité intitulé Methode pour apprendre de soimesme la choregraphie, ou l'art de decrire Et déchiffrer les Danses par caractères, Figures \& Signes démonstratifs publié par Dupré en 1757. Dans ce traité, il y a une partie explicative, puis des danses transcrites, dont L'entrée de l'Amiral de France (1756) que s'attribue l'auteur, et enfin un addenda, où cette danse apparaît cette fois sous forme de partition chorégraphique, en notation Beauchamp-Feuillet32.

Comme l'a montré Francine Lancelot, L'entrée de l'Amirale de France n'est pas en réalité une invention complète de Dupré, mais une version secondaire de L'entrée de Matelot attribuée à Feuillet, dont il existe une partition chorégraphique manuscrite probablement réalisée vers $1720^{33}$

Pour la présentation de ma démarche, je ne m'arrêterai que sur un micro exemple tiré à la fois de la transcription de danse et de la partition qui lui correspond. Cet exemple est un pas de la danse ; je l'ai choisi parce qu'il illustre un problème spécifique à la lecture analytique des sources pérennes de la danse.

Le pas en question se situe à la deuxième page de la partition, et à la vingt-et-unième mesure de la danse (Figure 2).

Illustration non autorisée à être diffusée

Figure 2 : Première page du mss "L'Entrée de l'Amiral de France (1756) », dans Dupré 1757. 
Nous le décryptons : le danseur, au départ du pas, se trouve en troisième position 34 le poids du corps réparti sur les deux pieds. Ses talons se touchent, et sont alignés l'un devant l'autre, pointes des pieds à quarante-cinq degrés.

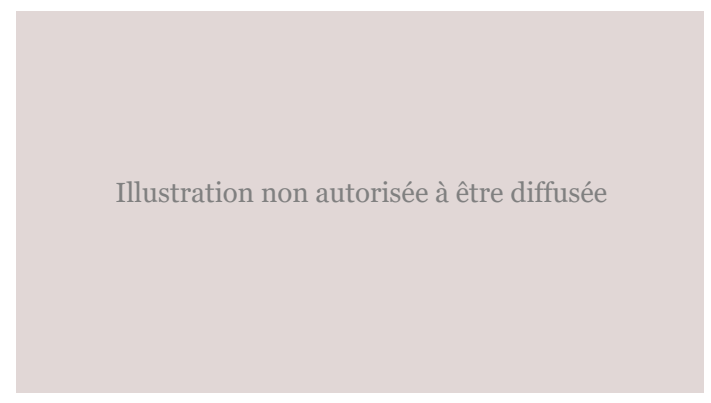

Figure 3 : Troisième position en notation Beauchamps-Feuillet, avec légendes.

Le danseur garde cette position tout au long du pas, pour ce qui est des pieds. Le pas s'exécute face au public35, et engendre un déplacement vers la droite.

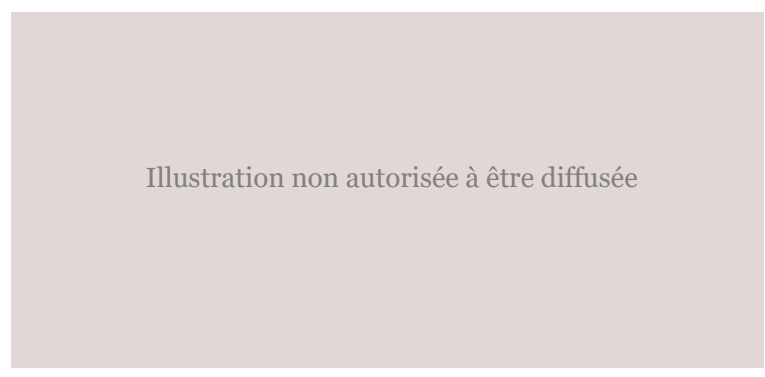

Figure 4 : Extrait de la deuxième page du mss "L'entrée de l'Amiral de France (1756)».

Toujours selon la notation, le pas se fait des deux pieds à la fois. On le déduit grâce à la liaison des deux têtes noires du $\operatorname{pas}^{36}$ (1)37. En observant le signe du plié (3) suivi de celui du sauté (3) sur les lignes incurvées qui marquent le déplacement du poids du corps (2), on comprend qu'il s'agit d'un saut fait des deux pieds.

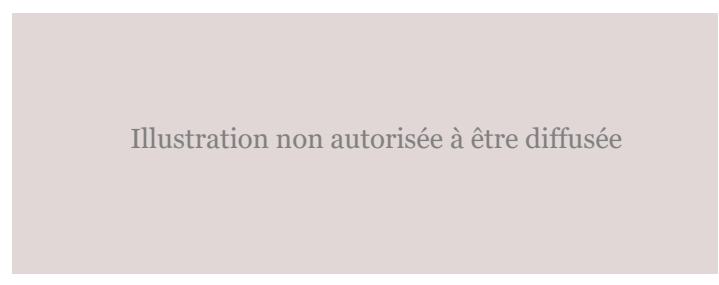

Figure 5 : Mesure chorégraphique 21 de "L'entrée de l'Amiral de France » avec légendes : 1: liaison des deux talons tels qu’ils sont représentés pour le départ du pas; 2: trait incurvé représentant le déplacement du poids du corps; 3: trait oblique représentant le déplacement du poids du corps; 3: trait oblique représentant le mouvement du plié; 4: deux traits perpendiculaire au trait incurvé, et parallèles entre eux, représentant le mouvement du saut.

En déchiffrant tous les éléments et en considérant leurs relations, on peut conclure donc que la $21^{\text {ème }}$ mesure de L'entrée de l'Amiral correspond à deux drôles de petits bons qui se font de côté vers la droite, qui débutent et s'achèvent par un plié des genoux. Ces bons se font en conservant la troisième position, pied droit derrière.

L'expérimentation de ce pas nous apprend deux choses : premièrement, la fin des sauts en plié implique une lourdeur dans l'exécution qui n'est pas conforme au style de la belle danse ; deuxièmement, la manière dont les pieds sont croisés pour le premier pas est pour ainsi dire anti-anatomique avec le déplacement du danseur vers sa droite. Pour pouvoir se déplacer assez tout en gardant son équilibre, précisément suite à l'expérimentation, nous proposons de placer son appui sur la jambe de derrière ce qui n'est pas habituel. Le plié sur l'atterrissage et le poids du corps sur le pied de derrière sont deux 
particularités respectivement représentées et déduite de la notation.

Deux choses en revanche restent obscures malgré la précision de cette notation. Premièrement c'est là la plus simple des questions à résoudre - , la position des pieds à l'arrivée du pas n'est pas nette ; elle ne correspond à aucune des codifications connues des positions des pieds sur lesquelles repose tout le vocabulaire de la « belle danse ». Or, ce pas paraît être en fait l'équivalent d'un syntagme qui comporte deux sauts. Sans être notés de manière identique, les deux sauts paraissent donc devoir être exécutés de la même façon. Cette hypothèse qui émane de l'incorporation de ce pas, mais aussi de l'habitude acquise par la lecture de la notation Beauchamps-Feuillet, se confirme ensuite dans la partition même, où un pas exécuté dans la direction opposée apparaît lors du « renversement » de la phrase chorégraphique.

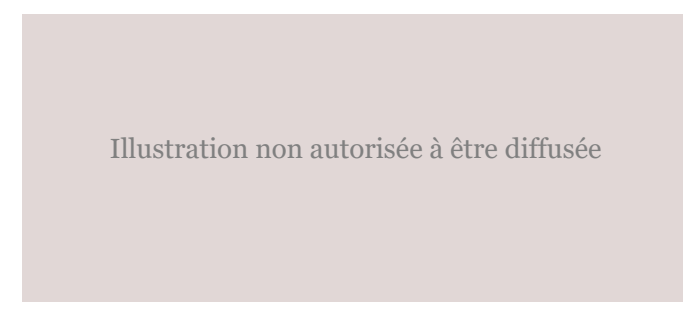

Figure 6 : Extrait de la deuxième page du mss "L'entrée de l'Amiral de France (1756)».

Cette version inversée du pas est sans ambiguïté quant à la position des pieds. Nous pouvons donc attribuer au notateur une imprécision graphique du pas, sans valeur expressive.

Deuxièmement, on peut se demander si ce pas, tel qu'il est déchiffré, a une signification kinesthésique dans le contexte du style de la belle danse, et si oui, laquelle. Cette question se pose à cause de l'effet de contraste que fait le pas dans la phrase chorégraphique où il apparaît. Pour y répondre, nous avons consulté des sources connexes.

Un extrait de La chorégraphie montre que le double bond de la mesure 21 n'est pas convenu parce qu'il n'est pas répertorié dans les tables des pas établies par Feuillet.

Illustration non autorisée à être diffusée

Figure 7 : Extrait de Feuillet 170o, p. 31.

Cette triple distinction entre le pas répertorié et le double bond de la mesure 21 atteste que ce dernier n'est pas représentatif du style de la belle danse. Il pourrait soit appartenir aux danses paysannes ou populaires - et ainsi soit illustrer un caractère marin de l'Entrée de l'Amiral de France soit être emprunté à ce vocabulaire à des fins parodiques.

L'existence exceptionnelle d'une description (ou transcription) de cette danse a laissé momentanément l'espoir de résoudre cette deuxième question ; mais cette description apporte un nouveau problème, pour autant qu'on soit adepte de la « théorie du doute ${ }^{38}$. Car ce texte propose un autre décodage de la notation : pour cette mesure chorégraphique, il est question de " chassés ». Or un chassé, en notation Beauchamp-Feuillet se note de cette manière : 


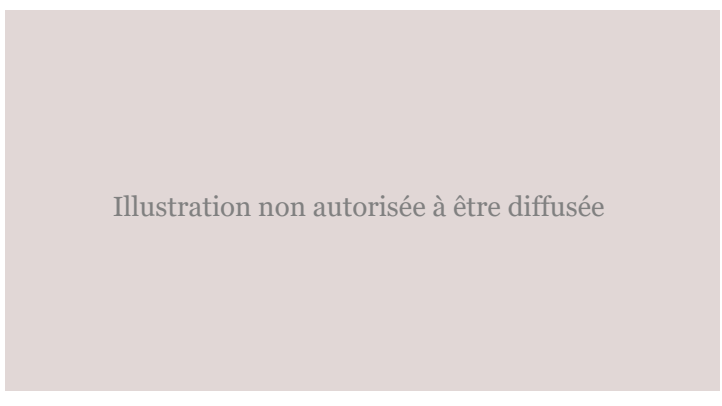

Figure 8 : Extrait de Feuillet 170o, p. 80.

L'expérimentation de cet autre pas met clairement en évidence deux choses.

La première est relative à la technique du pas et à son effet. Le chassé tel qu'il est défini à cette époque est très léger. Il se fait de cette manière : on part à la seconde position ${ }^{39}$, le poids en appui sur une seule jambe, puis on les plie sur la levée. Sur le premier temps, on se retrouver en l'air. Le chassé se répète toujours deux fois, ce qui permet au danseur de rebondir d'un pas à l'autre et de profiter de son élan.

La deuxième est relative à la syntaxe de la phrase chorégraphique dans laquelle le chassé est censé apparaitre. En fait, il existe des contraintes mécaniques qui théoriquement posent problème pour l'insertion du chassé dans la séquence de mouvement. Car avant le début des sauts, selon la partition chorégraphique et selon la déduction que l'on peut tirer de la transcription de la danse, on est en troisième position. Or, on devrait être en seconde position si l'on voulait effectivement faire des chassés. Cette deuxième observation exclut tout à fait que Dupré se soit livré à une simple transcription de la codification en notation Beauchamps-Feuillet, et qu'il se soit simplement trompé de nom. Il propose véritablement une autre solution, c'est-à-dire qu'il modifie la chorégraphie originale dont témoigne l'addenda.

Dupré, à mon avis, en remplaçant dans sa transcription un pas lourd d'exécution (le saut latéral) par un pas beaucoup plus gracieux (le chassé), poursuit l'anoblissement de la danse originale intitulée L'entrée de Matelot, «améliorée» et rebaptisée Entrée de l'Amiral de France. Aussi, nous sommes face à un cas où il ne peut pas être question seulement d'authenticité - la source que j'analyse elle-même ne l'est pas - mais seulement de cohérence interprétative, ce qui nous invite à faire valoir les deux possibilités de réalisation de la transcription et de la chorégraphie, sans les hiérarchiser.

L'expérimentation, dans la démarche analytique de ce pas, est décisive ${ }^{40}$. D'abord, elle permet d'identifier un problème, une question. Ensuite, des particularismes kinétiques sont révélés, qui sont porteurs d'information quant au style. Sur la base de ces informations, en complément avec d'autres sources, il est possible d'élaborer et d'étayer deux hypothèses interprétatives de la phrase chorégraphique, puis éventuellement de la pièce en général. Enfin, elle permet, après un contact avec des sources annexes, si ce n'est de départager parmi les hypothèses laquelle est pertinente et laquelle ne l'est pas, du moins de comprendre à quel point les implications de la modification d'un seul pas dans une phrase chorégraphique sont significatives.

\section{Limites de l'expérimentation}

D'un point de vue méthodologique, nos approches correspondent au schéma suivant : 
Figure 9: Schéma du processus interdisciplinaire incluant l'expérimentation.

Bien entendu, si l'on ne se fondait que sur l'expérimentation, cette méthode aurait beaucoup moins de potentiel que ce qu'elle a lorsqu'elle est inclue dans une démarche complexe composant des méthodes d'investigation et d'analyse conventionnelles pour la recherche fondamentale en sciences humaines. Elle s'inscrit donc nécessairement dans une démarche pluridisciplinaire et doit faire l'objet d'une attention particulière du point de vue méthodologique. Il est primordial dès le départ, mais également dans l'établissement des protocoles, ainsi que dans l'évaluation des résultats, de formaliser et de prendre en compte les limites de ce type de méthode. Il appartient à chaque expérimentateur de les définir et il serait difficile ici d'en dresser la liste exhaustive, néanmoins certaines limites communes se polarisent autour de :

- La corporalité : le corps de l'expérimentateur diffère de celui qui a fait référence pour l'objet d'investigation. Une des manières de contourner ce problème est la conduite des tests expérimentaux sur un large panel d'expérimentateurs et/ou de préparer le corps des expérimentateurs d'après l'étude de sources renseignant sur des aspects physiologiques (capacités spécifiques recherchées, régime, etc.) ;

-Le savoir sensorimoteur : ce point est articulé au premier, mais est lié spécifiquement aux types d'expériences, à la formation, ainsi qu'au profil du sujet investigué par rapport au type de geste codifié. 
Il est très difficile de pouvoir restreindre cette limite, puisque rares sont les textes techniques qui permettent d'identifier spécifiquement des individualités qui auraient laissé suffisamment de traces écrites pour permettre au chercheur de tester non seulement des gestes parfaits, mais aussi les exercices routiniers qui y préparent, ainsi que les éléments de son savoir sensorimoteur ;

- L'équipement : Le geste étudié est contraint ou au contraire rendu plus effectif par les vêtements portés et l'équipement spécifique ${ }^{41 .} \quad \mathrm{La}$ reconstruction de ces éléments est un point sensible. Les sources techniques ne mentionnent pas nécessairement en détail le type d'équipement nécessaire. Le processus de reconstruction de ces éléments, basé sur des études pluridisciplinaire également, comporte des compromis ${ }^{42}$ sur l'historicité de l'équipement. Il est essentiel de pouvoir mesurer l'influence kinétique de ces compromis sur les gestes expérimentés et les inclure dans l'évaluation ;

- L'évaluation contemporaine du geste : comme évoqué plus haut, il est essentiel de pouvoir établir une grille d'évaluation sur la base du peu d'informations disponibles, complétée par des hypothèses pour pouvoir approcher des critères contemporain d'évaluation du geste. Ce point est sensible puisqu'il fait appel à des notions subjectives.

\section{Conclusion}

Pour autant que ces limites soient identifiées, réduites dans la mesure du possible, prises en compte dans les différentes étapes de la méthode, mais surtout qu'elles ne représentent pas plus de zone d'ombres que les résultats n'apportent de lumière dans la problématique de l'approche pluridisciplinaire, l'utilisation de l'expérimentation est justifiée. D'ailleurs, telle que définie dans cet article, elle présente toutes les caractéristiques d'une démarche scientifique. Elle est issue d'un problème induit ou déduit par des données initiales (dépouillement des sources). Elle fait l'objet de l'établissement d'un protocole de tests. Ses résultats sont analysables et reproductibles.

Sans pouvoir prétendre qu'aucune autre manière d'aborder nos problèmes ne permettrait de parvenir à des résultats équivalents, nous sommes convaincus que la méthode expérimentale est très productive pour la lecture de sources directement en lien avec la réduction en art, mais également pour toutes autres sources qui convoquent la corporalité du lecteur, que cela soit explicitement ou non. Cette « production » n'est pas à penser comme un moyen de revenir au passé, de pouvoir le comprendre ou encore le restituer - ce qui est au moins en partie de l'ordre du leurre - , mais de développer une «attention» (pour reprendre le terme d'André Robinet) dont on ne peut anticiper toutes les implications, mais qui est très prometteuse pour la recherche en général 43 .

1 JAQUET Daniel, Combattre en armure à la fin du Moyen Âge et au début de la Renaissance d'après l'étude des livres de combat, thèse de doctorat dirigée par le professeur Franco Morenzoni, Université de Genève, 2013. KISS MÜTZENBERG Dora, La saisie du mouvement: de l'écriture et de la lecture des sources de la belle danse, thèse de doctorat dirigée par les professeurs Etienne Darbellay et Guillemette Bolens de l'Université de Genève et par Marina Nordera de l'Université Sophia Antipolis de Nice, 2013.

2 Par exemple: Bien que l'Art de la Danse ait toûjours esté reconnu l'un des plus honneste et plus necessaire à former le corps, et luy donner les premieres et plus naturelles dispositions à toute sorte d'exercice, et entre autres à ceux des armes; it par consequent d'un des plus avantageux est plus utiles à nostre Noblesse et autres qui ont l'honneur de nous approcher, non seulement en temps de guerre dans nos armées, mais mesme en temps de paix dans le divertissement de nos Ballets. Extrait de la Lettre patente, 1661, p. 3-4, citée dans KOUGIOUMTZOGLOUROUCHER Eugénia, La genèse du vocabulaire de la danse classique au XVIII ${ }^{e}$ siècle : "La belle dance» (1661-1701), vol. 3, 
thèse de doctorat, Ecole Pratique des Hautes Etudes, Paris, 1990, p. 63 .

3 Dans les traités de danse, on trouve en effet un certain nombre d'injonctions à l'exercice, comme cet exemple tiré du Maître à Danser de Rameau en 1725, "Seconde partie. De la manière de faire les bras avec les pas de Sissonne », p. 251 : « Je conseillerois volontiers à ceux qui sont curieux de faire les bras avec liberté, lorsqu'ils commencent à les faire, d'exercer aussi plusieurs de ces pas avec les bras, parce qu'outre que ces pas donnent de la legereté au corps, ils donnent aussi aux bras cette liberté. »; et l'on comprend que le corps du danseur étant malgré tout l'instrument sur lequel repose la danse, sa transformation a des implications sur l'idéal de la danse comme sur des réalisations bien particulières (qu'elles soient des interprétations ou des chorégraphies).

4 BoLENS Guilemette, Le style des gestes, Corporéité et kinésie dans le récit littéraire, Lausanne, Editions BHMS, 2008. Les citations ci-dessous sont tirées des pages 2, 17 et 19 .

5 ELIAS Norbert, Über den Prozeß der Zivilisation. Soziogenetische und psychogenetische Untersuchungen. Erster Band. Wandlungen des Verhaltens in den weltlichen Oberschichten des Abendlandes. Zweiter Band. Wandlungen der Gesellschaft. Entwurf einer Theorie der Zivilisation. Verlag Haus zum Falken, Basel, 1939.

$6 \mathrm{Ou}$ « intelligence kinesthésique », telle que définie en français dans la thèse de MERLEAU-PONTY Maurice, Phénoménologie de la perception, thèse de doctorat, Faculté des lettres de l'Université de Paris, NRF, 1945, p. 180.

7 MC CLELLAND John, " Présence littéraire de l'activité physique de la danse », KUSHNER Ellen (éd.), L'Époque de la Renaissance (1400-1600). Tome III: Maturations et mutations (1520-1560), John Benjamins Publishing Company, Amsterdam, 2011, p. 210-218

8 Cette fonction d'expression de la réalité intérieure de l'homme par la danse, quitte à améliorer l'âme par l'exercice du corps, n'est de loin pas nouvelle au XVIII ${ }^{e}$ siècle. Voir à ce propos SMITT Jean-Claude, "The rational of gestures in the West ", dans BREMER jan, RODENBURG Hermann (éds.), A Cultural History of Gesture, Ithaca and New-York, Cornell University Press, 1991 et en particulier p. 64-65: In the Middle Ages, gestures seemed to be the outer expression of the 'movements of the soul', whereas the 'discipline' of gestures (drawn for instance from a monastic rule) was supposed to improve the soul. A second axis consisted of what today we call non-verbal communication. [...] The third axis was concerned with efficacy, with its double meaning: the practical efficacy of technical gestures (sawing, moving, writing, etc.) and the symbolic efficacy of political of sacramental rituals.
9 À ce propos, je ne crois pas que l'on commence par se figurer le concept de noblesse avant de réaliser qu'elle a comme attribut la haute tenue de la tête. Informée par les recherches de Lakoff \& Johnson qui montrent à quel point « notre système conceptuel se développe [tout d'abord] par notre corps », c'est-à-dire par notre intelligence kinesthésique, je pense au contraire que l'on passe par la compréhension kinésique de la relation entre l'autre et soi pour évaluer relativement à soi la noblesse de l'autre, sa droiture, son degré d'éducation, et pour enrichir notre concept de la noblesse et notre représentation de nous-même par rapport à ce concept au cours de multiples expériences. Lire LAKOFF Georges and JOHNSON Mark, Philosophy in the Flesh, New York, Basic Books, 1999, par exemple p. 6 : Our conceptual systems are not totally relative and not merely a matter of historical contingency, even though a degree of conceptual relativity does exist and even though historical contingency does matter a great deal. [...] Because our conceptual systems grow out of our bodies, meaning is grounded in and through our bodies.

10 Nous renvoyons aux problématiques développées dans ANGLO Sydney, L'escrime, la danse et l'art de la guerre : le livre et la représentation du mouvement, Paris, Bibliothèque nationale de France, 2011, p. 4-11.

11 Ces documents ont fait l'objet d'un catalogue pour les sources issues d'espaces germanophones (constituant la large majorité du corpus) dans LENG Rainer, FRÜHMORGAN-VOSS Hella, OTT Norbert $H$. et al., Katalog der deutschsprachigen illustrierten Handschriften des Mittelalters Band 4/2, Lfg. 1/2: 38: 38. Fecht- und Ringbücher, C.H. Beck, München, 2009.

12 Ces traités sont de trois sortes : soit ils expliquent précisément la notation Beauchamps-Feuillet - dans ce cas ils reposent à la fois sur des gravures des composantes de cette notation et sur des textes didactiques - , soit ils se centrent sur la technique de la danse - et mélangent alors textes et gravures explicatives - , soit ils sont d'un type plus radicalement théorique, et élaborent un idéal de la danse sans en préciser l'incarnation - dans ce dernier cas, ils ne comportent pas d'illustrations.

13 Sur cette portée, on trouve une clef indispensable à la lecture des notes à la bonne tessiture, une indication du mètre qui précise tout à la fois la construction temporelle qui sous-tend la mélodie et le tempo - à un faible degré de précision - , et une " armure » qui permet de connaître la tonalité de la pièce musicale complète : des indications utiles au maitre à danser comme à l'exécutant de la danse.

14 Ce point apparait en filigrane à la lecture des sources, il est parfois explicité comme dans le premier témoin du corpus qui glose le poème de Liechtenauer. Voir à ce propos : JAQUET Daniel, "Entre éducation et festivité : Schirm- und Fechtschulen dans l'ancienne Confédération suisse, XVe-XVI 
siècles. ", dans MEUWLI Olivier (éd.), Le duel et le combat singulier en Suisse Romande: de l'Antiquité au XIXe siècle, Lausanne, Cabédita, 2012, p. 77-87.

15 MOLLOY Barry (éd.), The Cutting Edge: Studies in Ancient and Medieval Combat, Tempus, Stroud, 2007, p. 12.

16 Jean-Noël Laurenti distingue deux de ces perspectives qui ne sont pas scientifiques selon notre acception, même si elles relèvent aussi d'une forme d'expérimentation: " [...] Il faut distinguer deux types de démarches : d'une part celle, à valeur expérimentale, qui s'apparente à la restauration, qui tente de redonner une existence aussi pleine que possible à des objets vestiges du passé pour lesquels nous disposons de traces importantes, instruments, décors, costumes, chorégraphies notées, œuvres musicales ou scéniques fortement documentées, et cela sans perdre de vue le caractère nécessairement imparfait et provisoire de telle restauration ; d'autre part celles qui, s'inspirant des enseignements de la restauration, s'attaquent à des œuvres pour lesquelles on a conservé beaucoup moins d'éléments, ou bien entreprennent d'interpréter plus librement les œuvres fortement documentées, ou bien même visent à la création d'œuvres entièrement nouvelles. " LAURENTI JeanNoël, «En guise de présentation: Raisons d'un colloque, réflexions et bilans », Annales de l'Association pour un Centre de Recherche sur les Arts du Spectacle aux XVII e et XVIII e siècles, $\mathrm{n}^{\circ} 4,2010$, p. 8.

17 «Un phénomène frappe vos sens; vous l'observez avec l'intention d'en découvrir la cause, et pour cela, vous en supposez une dont vous cherchez la vérification en instituant une expérience. Le raisonnement suggéré par l'observation des phénomènes institue donc des expériences [...], et ce raisonnement constitue la méthode que j'appelle expérimentale, parce qu'en définitive l'expérience est le contrôle, le critérium de l'exactitude du raisonnement dans la recherche des causes ou de la vérité ». CHEVREUL Michèle-Eugène, Lettres adressées à $M$. Villemain sur la méthode en général, Garnier Frères, Paris, 1856.

18 Éléments bibliographiques clés examinés dans CARIOU JeanYves, « La formation de l'esprit scientifique-trois axes théoriques, un outil pratique : DiPHTeRIC », Bull. APBG, $\mathrm{n}^{\circ}$ 2, 2002, p. 279-320.

19 FRALEIGH Sondra-Horton, Dance and the lived body: a descriptive aesthetics, University of Pittsburg Press, Pittsburg, 1987.

20 GUISGAND Philippe, Les fils d'un entrelacs sans fin, la danse dans l'œuvre d'Anne Teresa De Keersmaeker, Presses Universitaires du Septentrion, Villeneuve d'Ascq, 2008, p. 10-11.
21 LENG Rainer et al 2009, , op. cit., p.23-34. Sur ce groupe de manuscrits voir: HILS Hans-Peter, « Gladiatoria: Über drei Fechthandschriften aus der ersten Hälfte des 15 Jahrhunderts. », Codices manuscripti, vol. 13, 1987, p. 1-54.

22 JAQUET Daniel, « Harnischfechten, une approche du duel en armure à pied d'après les traités de combat (XVe-XVI ${ }^{\mathrm{e}}$ siècles): élaboration d'une logique de combat ", dans COGNOT Fabrice (éd.), Arts de combat. Théorie \& Pratique en Europe-XIVe-XX siècle, A.E.D.E.H, Paris, 2011, p. 117-136.

23 Anonyme, Gladiatoria, 1425-1450 ; Kunsthistorisches Museum, Wien, KK5013.

24 Innerhalb, jnnerhalb, ausserhalb, auszerhalb. 21 occurencesoccurrences dans les 28 feuillets.

25 Wenn du Jm aber stichst Jnnerhalb seins swerts zu den gesicht So nymb sein tenke hant hinder den glid mit deiner tenken hant $\mathrm{zu}$ deine $<m>$ swert vnd schrait mit deine $<m>$ tenken fues Jnnerhalb seins tenken fues , vnd heb mit deine $<m>$ Swertt seine $<n>$ arm Rbersich, wann du Jm dartzü pracht hast So la var $<e>n$ die tenkche hant aus deim swert vnd greiff $\mathrm{Jm}$ damit reschlich vber den hals, vnd mit dein $<$ er $>$ rechten hant vass sein tenks diech Jnnerhalb So wirffestu Jn von dir als du oben gemalt sichst. Transcription par LORBEER Carsten, Transkription des Tunierhandbuchs KK5013, Pragmatische Schriftlichkeit, 2006 [en ligne : <http://www.pragmatischeschriftlichkeit.de/KK5013.html> (consulté le 21.12.2008)].

26 Halbschwert ou kurze schwert. Illustration de cette tenue sur l'illustration commentée. Commentaire sur cette tenue dans JAQUET Daniel, 2011, op . cit.,

27 Considéré du point de vue de l'expérimentateur faisant face à l'épée adverse.

$28 \mathrm{La}$ sélection des techniques est effectuée selon plusieurs filtres, mais de manière générale, correspond à l'ensemble des mentions textuelles et iconographiques des données du postulat. En l'occurrence pour ce manuscrit, le postulat cité a été testé sur quatorze techniques.

29 1. Mécanique: le geste est découvert et pratiqué sans équipement. 2. Avec équipement : la technique est exécutée avec équipement. 3. Dynamique: la technique est exécutée avec équipement avec intention (force, distance et efficacité) dans la situation de combat adéquate. 4. Mise en situation: les expérimentateurs équipés tentent de placer cette technique dans une séquence de combat non déterminée, avec intention.

30 En définissant le cadre d'application des techniques, il est nécessaire d'en faire l'étude correspondant à la période et à l'espace géographique de la source. Idéalement, il faut également 
que cette étude soit effectuée à partir de plusieurs sources différentes (documents normatifs, littérature technique, etc.).

31 Ce critère fait appel au domaine du sensible des expérimentateurs. Il faut toujours analyser et tenter de définir les causes observables de la gêne, de manière à déterminer si elles sont induites par un mauvais usage de l'équipement, un simulateur dont les propriétés inhérentes font l'objet de limitation ou encore un problème lié à la corporalité. En effet, de nombreux mouvements martiaux sont par définition contraignants pour le corps.

32 Bien qu'on n'ait aucune source primaire des travaux de Beauchamps, on préfère aujourd'hui lui restituer une partie de l'invention de la notation que Feuillet s'est attribuée par la publication de La Chorégraphie en 1700.

33 Voir LANCELOT Francine (éd.), La Belle Danse, catalogue raisonné fait en l'An 1995, Van Dieren Éditeur, Paris, 1996., p. 301.

34 Le signe représentant la troisième position est formé par les deux ronds dont partent deux traits formant ensemble un angle de quarante-cinq degrés.

35 L'orientation du signe des pieds se déduit relativement à la page, dont le haut correspond à la place du public, permet de le déduire.

36 Cette liaison se répète deux fois : la première se situe dès après la petite barre de mesure perpendiculaire à la longue ligne qui désigne ici le trajet que fait le danseur ; la seconde peut être observée après le premier signe de position où les ronds évidés représentent les talons du danseur.

37 La numérotation, dans ce paragraphe, renvoie au croquis avec légende de la mesure chorégraphique 21 de « L'entrée de l'Amiral de France ".

38 "Ce que j'appelle la " pratique du doute " et qui, à mon sens, spécifie les études de genre, relève aussi de la méthode critique. Elle consiste à mettre en questions, de façon systématique le récit historique tel qu'il est présenté, les "évidences" culturelles, et aussi les convictions et les représentations de la chercheuse et du chercheur. Ce doute n'est pas que critique, il fonde d'autres questionnements, d'autres approches, ouvre de nouvelles perspectives. » MARQUIÉ Hélène, «Le genre, un outil épistémologique pour l'historiographie de la danse » dans Les Arts de la scène à l'épreuve de l'histoire: Les objets et les méthodes de l'historiographie des spectacles produits sur la scène française (1635-1906), Champion, Paris, 2011, p. 216.

39 La seconde position est l'écartement des deux pieds à distance de 25 à 35 centimètres ; on obtient donc une position très stable où les pieds sont presque sous les épaules.
40 Il existe un sentiment partagé d'ivresse et de désarroi face à de tels cas, qui a été exprimé d'une manière aussi auto-ironique que réaliste en ces terme: " nous sommes [les chercheurs œuvrant pour la compréhension des arts du spectacle du XVII et XVIII e siècle] à vrai dire dans la situation du juge chargé de l'instruction d'un délit pour lequel aucun retour sur le fait n'est possible car il n'a aucun film d'amateur, aucune vidéo de caméra de surveillance. Il lui faut donc confronter des indices et des témoignages. Il n'accédera plus jamais au réel, mais il peut au mieux modéliser de façon conjecturale l'image-robot de la chose. » CARON Philippe, « La diction composite de la comédie. À propos du Bourgeois gentilhomme », Annales de l'Association pour un Centre de Recherche sur les Arts du Spectacle aux XVII ${ }^{e}$ et $X V I I I^{e}$ siècles, $\mathrm{n}^{\circ} 4$, 2010, p. 185.

41 Il est souvent fait mention de «costume». Nous utilisons délibérément "vêtement ", puisque les pièces d'habillement/accessoires qui étaient portés pour réaliser les gestes doivent faire l'objet de recherches très précises de manière à pouvoir être reconstruites au plus proche possible des originaux (pièce conservée ou étude à partir de plusieurs sources iconographiques). Il ne s'agit donc pas de costume, mais bien de vêtements ou d'équipements fonctionnels, conçus (taille, technique, étoffe/matériaux) pour être effectif et non pour simuler ou ressembler à des vêtements d'époque.

42 Les techniques de fabrication (découpe, assemblage, forge, etc.), les outils ou les procédés utilisés (couture à la main, réduction de minerai de fer en plaque d'acier, tissage, travail sur le cuir), ainsi que les matériaux spécifiques utilisés ne sont pas aisément reproductibles (disponibilité, coût ou manque d'informations dans les sources). Ils doivent faire l'objet de compromis dans la reconstruction. Il est essentiel pour l'expérimentateur de s'entourer de spécialistes qui pourront lui prodiguer des conseils sur la base d'études spécifiques.

43 " "L'attention " n'est pas une " activité " générale et formelle, mais une manifestation de l'actualité perceptive qui s'oriente dans son propre espace expérimental, qui réalise de nouvelles articulations, découpant l'horizon de monde et constituant des régions neuves. La vraie théorie de l'attention n'est donc pas au terme de l'emploi de l'induction ou de la déduction, mais de la mise en présence du principe du réfléchissant et de l'irréfléchi, dans l'éveil du sujet à sa propre histoire qu'il oubliait. » ROBINET André, Merleau-Ponty, sa vie, son œuvre, Presses Universitaires de France, Paris, 1963, p. 16. 